\title{
Photodynamic therapy with hexenyl ester of 5-aminolevulinic acid induces necrotic cell death in salivary gland adenocarcinoma cells
}

\author{
JONG-HWAN PARK ${ }^{1}$, YEON-HEE MOON ${ }^{2}$, DONG-JAE KIM ${ }^{2}$, SOO-A KIM ${ }^{3}$, \\ JEE-BUM LEE ${ }^{4}$, SANG-GUN AHN ${ }^{2}$ and JUNG-HOON YOON ${ }^{2}$ \\ ${ }^{1}$ Department of Biochemistry, College of Medicine, Konyang University, Daejon 302-718; ${ }^{2}$ Department of Pathology, \\ School of Dentistry, Chosun University, Gwangju 501-759; ${ }^{3}$ Department of Biochemistry, \\ College of Oriental Medicine, Dongguk University, Gyungju 780-714; ${ }^{4}$ Department of Dermatology, \\ Chonnam National University Medical School, 8-Hak-dong, Dong-gu, Gwangju 501-757, Korea
}

Received January 28, 2010; Accepted March 26, 2010

DOI: $10.3892 /$ or_00000843

\begin{abstract}
Photodynamic therapy has been developed as an alternative therapy of cancer. The aim of this study was to examine whether PDT with hexenyl ester of 5-aminolaevulinic acid (ALA-hx) inhibits the proliferation of the salivary gland adenocarcinoma SGT cells. Cell proliferation was examined by MTT assay. The gene expression of Coproporphyrinogen oxidase (CPO) and ROS production was also examined. Flow cytometry and in vivo Chorioallantoic membrane (CAM) assay was performed. ALA-hx PDT inhibited effectively the proliferation of SGT cells. Treatment of ALAhx induced CPO mRNA expression and ROS was produced by ALA-hx PDT in SGT cells. Flow cytometry and LDH assay showed that ALA-hx PDT induced necrotic cell death rather than apoptosis in SGT cells. In vivo CAM assay showed that ALA-hx PDT induced tumor destruction by inducing necrosis. These results indicate that ALA-hx PDT effectively inhibits the proliferation of SGT cells by inducing necrosis.
\end{abstract}

\section{Introduction}

Salivary gland cancers account for $\sim 3-6 \%$ of all head and neck tumors in adults (1-3). Salivary gland tumor can be classified according to histological characteristics and biological behavior (2). The WHO classification of malignant salivary gland tumors has been recently updated, listing 24 different histologic subtypes (4). The main four histopathologic types include mucoepidermoid carcinoma (29-34\% of malignant tumors), adenoid cystic carcinoma ( 20\%), adenocarcinoma, and salivary duct carcinoma.

Correspondence to: Dr Jung-Hoon Yoon or Dr Sang-Gun Ahn, Department of Pathology, School of Dentistry, Chosun University, 375 Seosuk-dong, Dong-gu, Gwangju 501-759, Korea E-mail: jhyoon@chosun.ac.kr or ahnsg@chosun.ac.kr

Key words: photodynamic therapy, hexenyl ester of 5-aminolevulinic acid, salivary gland adenocarcinoma
Salivary gland carcinomas are primarily managed with surgical resection, often in conjunction with postoperative radiation therapy (5). The functions of chemotherapy in the management of salivary gland cancers remain unsolved (2). A recent study showed that response rates of salivary gland cancers to chemotherapy are inconsistent, varied and generally poor (6). On account of this, chemotherapy is generally indicated only for palliation in symptomatic patients with recurrent and/or unresectable cancers (5) and the development of alternative therapy is necessary.

Photodynamic therapy (PDT) with 5-aminolaevulinic acid (ALA) has been widely investigated for the treatment of cancer (7). ALA and its esters act as precursors to the photosensitizer protoporphyrin IX (PpIX) $(8,9)$. ALA serves as the biological precursor in the heme biosynthesis pathway (8-11). Regulatory feedback system in heme biosynthesis is bypassed by adding exogenous ALA, which lead to the accumulation of PpIX in cells (12). Upon light exposure, PpIX is converted into its excited triplet state, enabling energy transfer to ground state molecular oxygen, which is then converted to reactive singlet oxygen. The latter has a capability to cause cell death such as necrosis or apoptosis (13).

As a doubly charged molecule, ALA does not penetrate easily across cell membranes (14). To overcome the limit of uptake and distribution of ALA, the existing drug has been converted into its esters to increase its lipophilic nature (15-17). Several ALA esters has been synthesized and investigated as promising precursors to PpIX in vitro and in vivo as well (15). The esterified ALA derivatives significantly increase PpIX levels over ALA in a variety of cell types (12-17).

We have previously demonstrated that ALA-hexenyl ester (ALA-hx), as an unsaturated alkyl ester, increased the PpIX accumulation in cell compared to other esters of ALA in a broad range of concentrations (18). Additionally, PDT using ALA-hx (ALA-hx PDT) significantly suppressed the tumor cell proliferation and induced cell death in vitro $(18,19)$. In this study, we examined anti-cancer effect of ALA-hx PDT in the SGT salivary gland tumor cell line. 


\section{Materials and methods}

Cell culture and reagents. SGT human salivary gland adenocarcinoma cells (20) (a kind gift from Professor C.H. Lee, Dankook University) were cultured in Dulbecco's modified Eagle's medium (DMEM) supplemented with $10 \%$ fetal bovine serum, $100 \mu \mathrm{g} / \mathrm{ml}$ streptomycin and $100 \mathrm{U} / \mathrm{ml}$ penicillin at $37^{\circ} \mathrm{C}$ in a $5 \% \mathrm{CO}_{2}$ incubator. ALA was purchased from Sigma (St. Louis, MO, USA). The ALA hexenyl ester $(\mathrm{C} 11 \mathrm{H} 19 \mathrm{NO} \cdot \mathrm{HCl}, \mathrm{ALA}-\mathrm{hx})$ were made as previously described (18). ALA and ALA-hx were dissolved in serumfree RPMI medium immediately before use.

Photodynamic treatment. SGT cells were washed with PBS and incubated in serum-free culture medium containing each photosensitizer of ALA and ALA-hx. Four hours after photosensitizer administration, the cells were exposed with light doses of $5 \mathrm{~J} / \mathrm{cm}^{2}$ of a red light-emitting diode (LED) light with 613-645 nm wavelength, with peak $635 \mathrm{~nm}$ (Philips Luxeon Lumileds, San Jose, CA, USA) at $35 \mathrm{~mW} / \mathrm{cm}^{2}$ as measured with a Delta Ohm DO 9721 quantum photo-radiometer and thermometer data logger (model DO9721, Padua, Italy) (18).

MTT assay. At 12 and $24 \mathrm{~h}$ after PDT, the cell viability was assessed using MTT assay. Briefly, the cells were washed twice with ice-cold PBS, and $0.5 \mathrm{ml}$ of cell culture medium and $50 \mu 1$ of 3-(4,5-dimethylthiazol-2-yl)-2, 5-diphenyltetrazolium bromide reagent $(5 \mathrm{mg} / \mathrm{ml}$ in PBS) were added and incubated for $3 \mathrm{~h}$ at $37^{\circ} \mathrm{C}$. The media were then removed and $250 \mu \mathrm{l}$ of acid-isopropanol $(0.04 \mathrm{~mol} / \mathrm{l} \mathrm{HCl}$ in isopropanol $)$ were added. The optical density (OD) was then measured by a microplate autoreader ELISA (Bio-Tek Instruments Inc., Winooski, VT) at $570 \mathrm{~nm}$ wavelength.

RT-PCR analysis for coproporphyrinogen oxidase (CPO). Total RNA was harvested from cells with TRIzol reagent (Invitrogen) as described by the manufacturer. Total RNA $(1 \mu \mathrm{g})$ was reverse transcribed into cDNA and PCR was performed using the Superscript ${ }^{\mathrm{TM}}$ One-step RT-PCR with platinum ${ }^{\circledR}$ Taq kit (Invitrogen). The following primer sets were used: CPO; F: 5'-CTCCTACTATCCATTTCAACTA CAG-3', R: 5'-CACGATGGGCTATAAAGAAGTAATC-3'. GAPDH; F: 5'-CCAAGGTCATCCATGACAACTTTG-3', R: 5'-GTCATACCAGGAAATGAGCTTGACA-3'. PCR conditions were: $50^{\circ} \mathrm{C}, 30 \mathrm{~min} ; 94^{\circ} \mathrm{C}, 30 \mathrm{sec} ; 55^{\circ} \mathrm{C}, 30 \mathrm{sec}$; $72^{\circ} \mathrm{C}, 30 \mathrm{sec} \times 29$ cycles; and $72^{\circ} \mathrm{C}, 10 \mathrm{~min}$. PCR products were then electrophoresed on a $5 \%$ acrylamide gel, visualized, and photographed using a gel documentation system.

Measurement of intracellular ROS. After PDT, the cells were washed twice with PBS and incubated in medium containing $10 \mu \mathrm{M} 2^{\prime} 7^{\prime}$-dichlorofluorescein diacetate, $\mathrm{H}_{2}$ DCFDA (Molecular Probes ${ }^{\mathrm{TM}}$, Eugene, OR, USA) at $37^{\circ} \mathrm{C}$ in an atmosphere containing $5 \% \mathrm{CO}_{2}$ for $30 \mathrm{~min}$. Fluorescence was analyzed immediately by Thermo Scientific Varioskan ${ }^{\circledR}$ Flash spectral scanning multimode reader (Varioskan, Thermo Electron Co., Waltham, MA, USA) with SkanIt ${ }^{\circledR}$ Software (Wavelength: Excitation 450-495, Emission 517-527).
Flow cytometry. At $24 \mathrm{~h}$ after PDT, the cells were stained with Alexa Fluor 488 Annexin V and propidium iodide (Vybrant apoptosis assay kit, Molecular Probes), according to the manufacturer's protocol. After incubated for $15 \mathrm{~min}$ in the darkness at room temperature, the $1 \times 10^{4}$ cells per sample were analyzed immediately using Cell Llab Quanta ${ }^{\mathrm{TM}} \mathrm{SC}$ flow cytometer (Beckman Coulter Inc., Miami, FL, USA) and software.

LDH assay. LDH was measured from culture supernatant using commercial kit according to manufacturer's instructions (Promega, Madison, WI, USA).

Chorioallantoic membrane (CAM) assay. The fertilized chicken eggs (Yangsung, Gyeonggi, Korea) were kept in a humidified incubator (VS-9160, Vision Science, Bucheon, Korea) at $37^{\circ} \mathrm{C}$. At day 10 of incubation, the eggs were candled and wiped with $70 \%$ ethanol. The portion of eggshell above the air-sac was removed. To avoid capillary bleeding, pre-warmed PBS was placed on the shell membrane for $3 \mathrm{~min}$. CAM was exposed carefully by removing shell membrane. SGT cells $\left(2 \times 10^{6}\right)$ in $20 \mu 1$ of serum-free culture medium were placed directly on CAM. After 3 days, $10 \mu \mathrm{M}$ ALA-hx was applied to the tumor and subsequently $4 \mathrm{~h}$ later, $10 \mathrm{~J} / \mathrm{cm}^{2}$ LED was given for $6 \mathrm{~min}$. The removed eggshell portion was closed by cellophane tape and eggs were returned to incubator. After $24 \mathrm{~h}$ incubation, CAM was obtained from eggs and fixed $10 \%$ neutral formalin for $24 \mathrm{~h}$. Fixed CAM was dehydrated in an alcohol-xylene series, and embedded in paraffin wax. From each block, $2 \mu \mathrm{m}$ sections were prepared and stained with hematoxylin and eosin for histopathological examination.

\section{Results}

As shown in Fig. 1A, ALA-hx PDT effectively reduced the viability of SGT cells, which was dependent on dose of ALA$\mathrm{hx}$. In addition, the inhibitory effect was more significant at $24 \mathrm{~h}$ after PDT (Fig. 1B).

ALA and its esters have been used as precursors to the fluorescent photosensitizer protoporphyrin IX (PpIX) and $\mathrm{CPO}$, a rate-limiting enzyme, is involved in the regulation of PpIX production (21). Therefore, we examined whether ALA-hx affects the gene expression of CPO. ALA-hx upregulated mRNA expression of CPO in SGT cells in a dose-dependent manner (Fig. 2A and B).

Since ROS is critical for cell death induced by PDT, we examined ROS production by ALA-hx PDT in SGT cells. Fluorescent microscopic photographs showed that ALA-hx PDT led to intracellular ROS production at $1 \mathrm{~h}$ after PDT (Fig. 3A). When quantified using the scanning multimode reader and software, only ALA-hx PDT significantly induced ROS production, whereas the photosensitizer (ALA-hx) or LED alone did not (Fig. 3B).

To clarify whether cell death by ALA-hx PDT in SGT cells is due to apoptosis or necrosis, flow cytometry was performed. LED or ALA-hx alone did not affect the ratio of PI- or annexin V-positive cells (Fig. 4A). However, ALA-hx PDT markedly increased the ratio of PI-positive cells, but did not affect annexin V staining. In addition, ALA-hx PDT induced LDH secretion in SGT cells (Fig. 4B). These results 

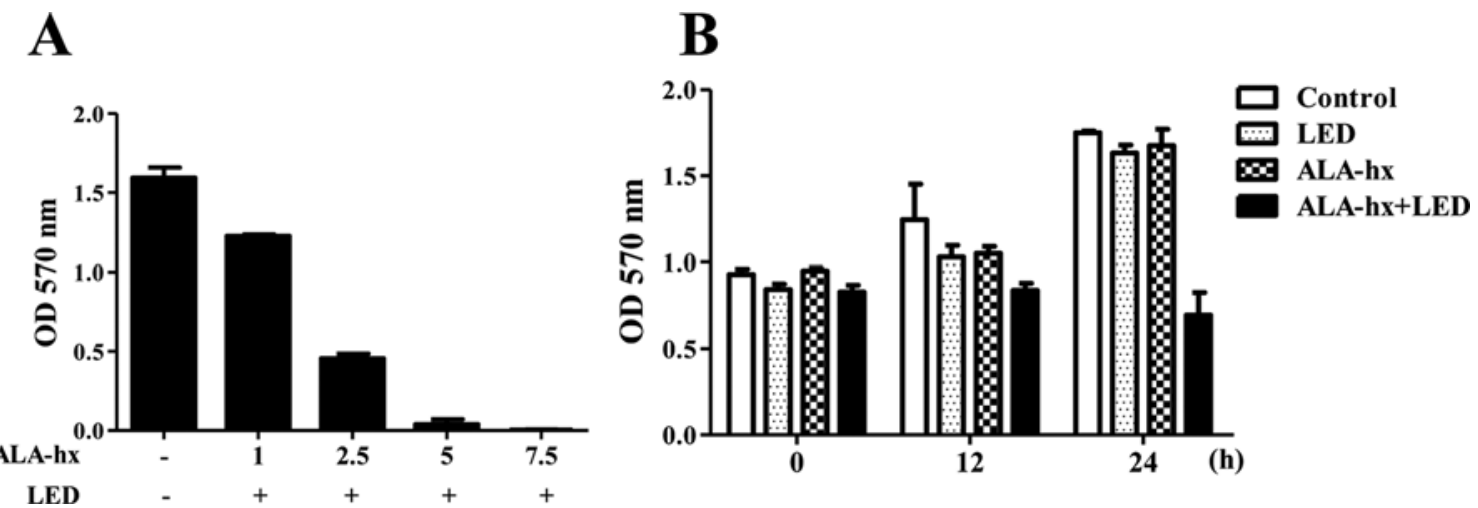

Figure 1. Cell viability after ALA-hx PDT in SGT cells. (A) The cells were incubated with various doses of ALA-hx in serum-free medium for $4 \mathrm{~h}$ and exposed with $5 \mathrm{~J} / \mathrm{cm}^{2}$ of LED. After $24 \mathrm{~h}$, cell viability was examined by MTT assay. (B) The cells were incubated with $2.5 \mu \mathrm{M}$ of ALA-hx and exposed with $5 \mathrm{~J} / \mathrm{cm}^{2}$ of LED. After 12 and $24 \mathrm{~h}$, cell viability was examined.

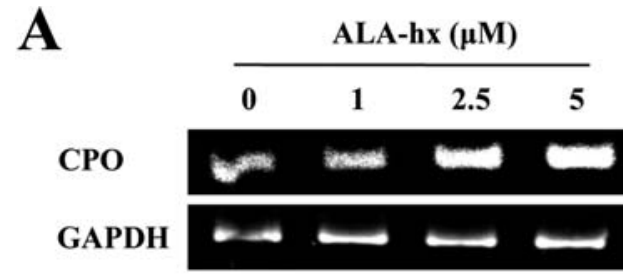

B

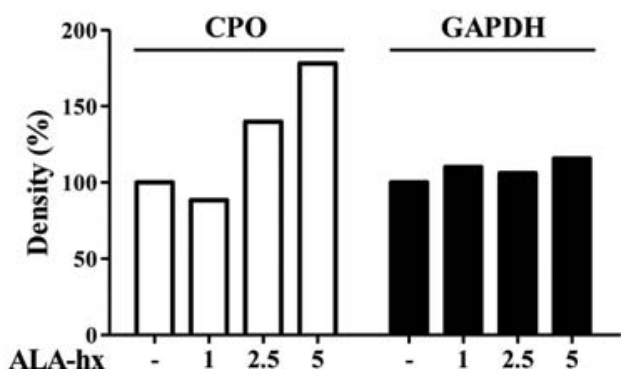

Figure 2. mRNA expression of CPO after ALA-hx treatment in SGT cells. (A) The cells were treated with different doses of ALA-hx and $4 \mathrm{~h}$ later, CPO mRNA expression was examined by RT-PCR. (B) The band density was expressed as percentage vs. that of control.

indicate that ALA-hx PDT induces necrotic cell death in SGT cells.

Histopathological examination showed that inoculated SGT cells were adhered to surface of CAM at 3 days after inoculation. At this time, invasion of CAM by tumor cells were not detected. The layer of proliferating cells was clearly separated from endoderm of CAM by ectoderm barrier (Fig. 5A). Application of PDT clearly demonstrated necrotic cell death of SGT cells. Tumor cells with eosinophilic cytoplasm and necrotic body were observed in PDT applied CAM (Fig. 5B).

\section{Discussion}

Surgical resection, with adjuvant radiation, is primarily applied for salivary gland cancer (22). Although systemic chemotherapy has been used for recurrence or metastatic diseases, the response rate is low with the range of $15-50 \%$
A

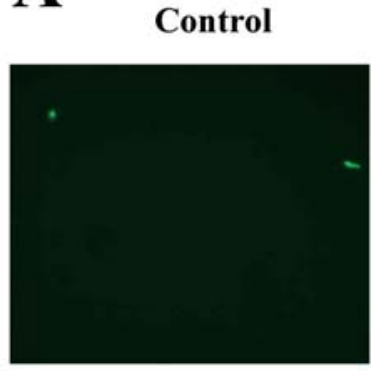

ALA-hx + LED

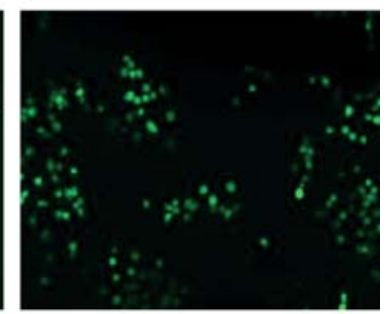

B

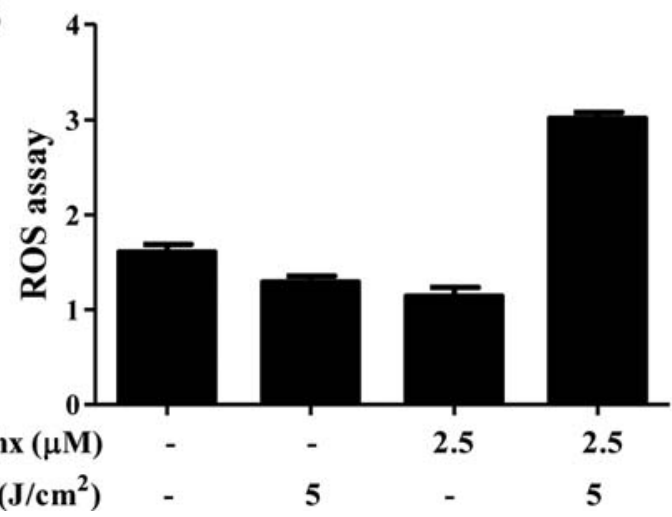

Figure 3. ROS production in SGT cells with ALA-hx PDT. The cells were incubated with $2.5 \mu \mathrm{M}$ ALA-hx followed by exposure to $5 \mathrm{~J} / \mathrm{cm}^{2}$ of laser light. (A) The images were obtained by fluorescent microscopy at $1 \mathrm{~h}$ after PDT and (B) quantitative amount of ROS production was measured using the scanning multimode reader and software.

(23). Because of disadvantages of current treatment such as side effects, toxicity, or low response late, there is a need to develop alternative therapy. PDT is currently being used as a therapeutic alternative for a variety of malignant tumors. Compared with ionizing radiation therapy or chemotherapy, PDT is generally safer for the surrounding normal tissues because photosensitizers are preferentially accumulated in tumor cells (24). PDT is based on the interaction of a photosensitizer and visible light with a suitable wavelength which causes the destruction of cancer cells by the formation of reactive oxygen species (ROS) (10). Among esters of ALA, 
A

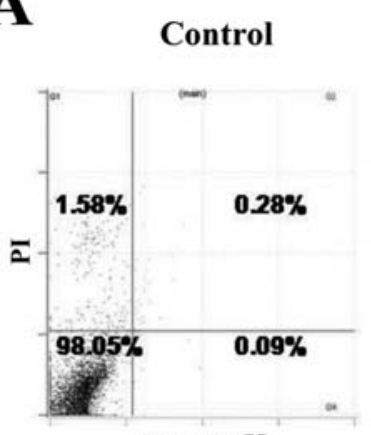

Annexin V

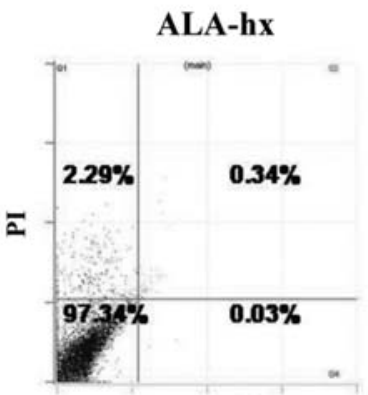

Annexin V
LED
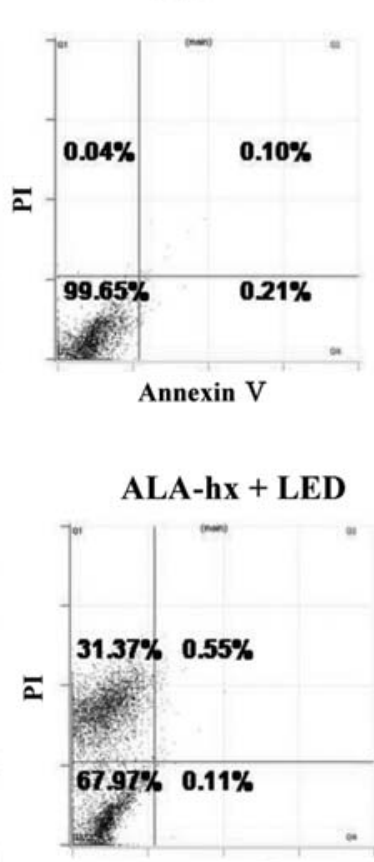

Annexin V

\section{Control}

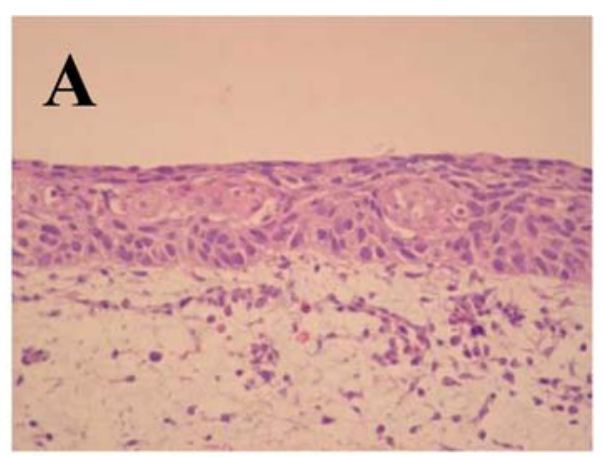

\section{ALA-hx

+ LED

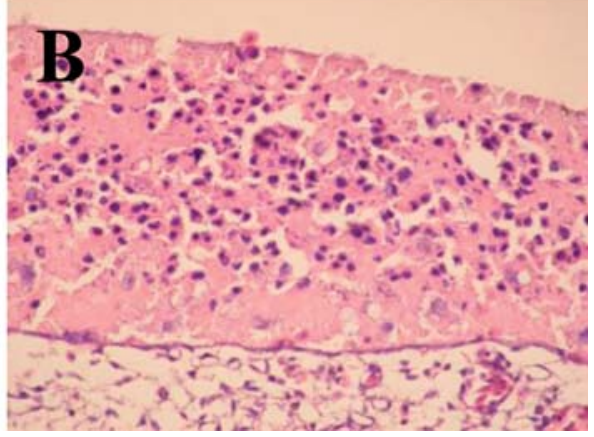

Figure 5. In vivo CAM assay. CAM assay was performed as described in Materials and methods. Histopathological findings were obtained from H\&E stained sections. Control (A); ALA-hx PDT treated (B).

ALA-hx induced more PpIX synthesis in A431 cells than other esters of ALA (18). In our previous study, ALA-hx PDT was found to have more effective anti-cancer property in YD-10B cells, a human oral squamous cell carcinoma, than ALA-mediated PDT (19). The present study was designed to examine whether ALA-hx PDT is clinically applicable to salivary gland cancers.
B

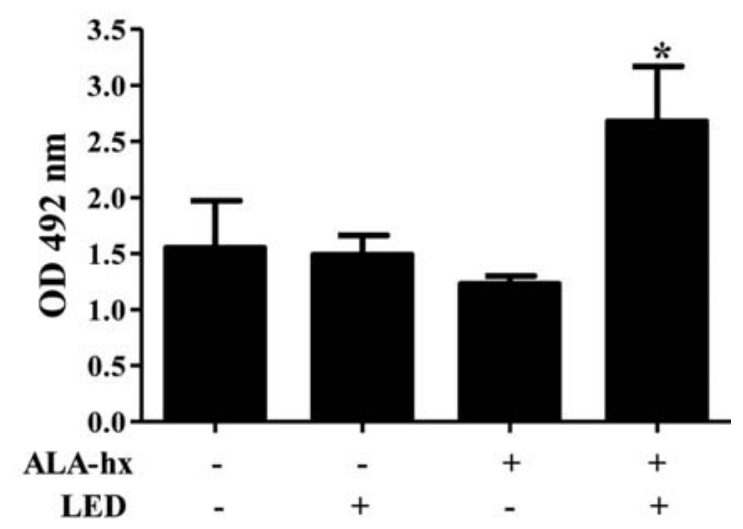

Figure 4. Flow cytometry analysis and LDH measurement. The cells were incubated with $2.5 \mu \mathrm{M}$ of ALA-hx for $4 \mathrm{~h}$ followed by exposure to $5 \mathrm{~J} / \mathrm{cm}^{2}$ of LED. (A) Subsequently, flow cytometric analysis was performed using annexin $\mathrm{V}$ and PI staining. (B) In addition, LDH was measured from culture supernatant. ${ }^{*} \mathrm{P}<0.05$

PDT inhibits the cell proliferation by inducing apoptosis or necrosis. Therefore, we examined the effect of ALA-hx PDT on the viability of SGT cells. MTT assay showed that ALA-hx PDT inhibited effectively the growth of SGT cells in a dose-dependent manner. ALA and its esters act as precursors of the photosensitizer PpIX and CPO regulates the PpIX synthesis. In this study, the gene expression of CPO was enhanced by ALA-hx in SGT cells, suggesting that ALA-hx may effectively induce PpIX synthesis in SGT cells. In addition, production of ROS by PDT leads to damage of the cellular constituents and subsequent cell death $(10,25)$. In this study, ALA-hx PDT increased significantly ROS production in SGT cells, even at a concentration of $2.5 \mu \mathrm{M}$ of ALA-hx, a relatively low dose. Collectively, our results showed that ALA-hx PDT effectively suppressed the proliferation of SGT cells by inducing CPO gene expression and ROS production.

Although PDT induced cell death in a variety of cancers, the molecular mechanisms involved in each death event might be different $(26,27)$. It is increasingly considered that apoptosis is involved in PDT-mediated tumor cell death in vitro and tumor ablation in vivo $(26,27)$. Our previous study revealed that ALA-hx PDT caused apoptosis in YD-10B cells through mitochondrial-dependent pathway (19). However, in this study, ALA-hx PDT induced necrotic cell death of SGT cells, suggesting that the mechanism of ALA-hx PDT-induced cell death may be cell type-specific.

CAM assay has been used as a valuable model system for in vivo studies on tumor development, angiogenesis, and metastasis. Highly vascularized CAM provides an enriched environment for rapid tumor formation and angiogenesis following cell grafting. In addition, embryo is immunoincompetent until day 18 of incubation so the immune rejection against grafted cells does not occur. In our study, we evaluated anti-tumor effect of ALA-hx PDT using in vivo simulating 
CAM assay model. ALA-hx PDT led to tumor destruction by inducing necrotic cell death, suggesting that ALA-hx PDT may be applicable to salivary gland cancers clinically.

In summary, in vitro study showed that ALA-hx PDT led to the production of ROS in SGT cells and effectively inhibited the cell growth. Flow cytometry and LDH assay showed ALA-hx PDT-induced cell death of SGT cells was due to necrosis. In vivo CAM assay, histopathological findings also revealed that ALA-hx PDT induced necrosis of the tumor. These findings suggest that ALA-hx PDT can be used as a therapeutic alternative for salivary gland cancers.

\section{Acknowledgements}

This study was supported from Korea Science and Engineering Foundation (KOSEF) grant funded by the Korea government (MOST) (No. R13-2008-010-01001-0).

\section{References}

1. Etges A, Nunes FD, Ribeiro KC and Araujo VC: Immunohistochemical expression of retinoblastoma pathway proteins in normal salivary glands and in salivary gland tumours. Oral Oncol 40: 326-331, 2004.

2. Milano A, Longo F, Basile M, Iaffaioli RV and Caponigro F: Recent advances in the treatment of salivary gland cancers: emphasis on molecular targeted therapy. Oral Oncol 43: 729-734, 2007.

3. Prenen H, Kimpe M and Nuyts S: Salivary gland carcinomas: molecular abnormalities as potential therapeutic targets. Curr Opin Oncol 20: 270-274, 2008

4. Barnes L, Everson JW and Reichart P: World Health Organization classification of tumours. IARC Press, Lyon, 2005.

5. Spiro RH: Management of malignant tumors of the salivary glands. Oncology 12: 671-683, 1998.

6. Dodd RL and Slevin NJ: Salivary gland adenoid cystic carcinoma: a review of chemotherapy and molecular therapies. Oral Oncol 42: 759-769, 2006

7. Kelty CJ, Brown NJ, Reed MW and Ackroyd R: The use of 5-aminolaevulinic acid as a photosensitiser in photodynamic therapy and photodiagnosis. Photochem Photobiol Sci 1: 158-168, 2002 .

8. Sharma S, Jajoo A and Dube A: 5-Aminolevulinic acid-induced protoporphyrin-IX accumulation and associated phototoxicity in macrophages and oral cancer cell lines. J Photochem Photobiol B 88: 156-162, 2007

9. van den Akker JT, Iani V, Star WM, Sterenborg HJ and Moan J: Topical application of 5-aminolevulinic acid hexyl ester and 5-aminolevulinic acid to normal nude mouse skin: differences in protoporphyrin IX fluorescence kinetics and the role of the stratum corneum. Photochem Photobiol 72: 681-689, 2000.

10. Castano AP, Demidova TN and Hamblin MR: Mechanisms in photodynamic therapy: part one-photosensitizers, photochemistry and cellular localization. Photodiagnosis Photodyn Ther 1: 279-293, 2004

11. Tsai T, Hong RL, Tsai JC, Lou PJ, Ling IF and Chen CT: Effect of 5-aminolevulinic acid-mediated photodynamic therapy on MCF-7 and MCF-7/ADR cells. Lasers Surg Med 34: 62-72, 2004.
12. Wu RW, Chu ES, Yow CM and Chen JY: Photodynamic effects on nasopharyngeal carcinoma (NPC) cells with 5-aminolevulinic acid or its hexyl ester. Cancer Lett 242: 112-119, 2006.

13. Gerscher S, Connelly JP, Griffiths J, et al: Comparison of the pharmacokinetics and phototoxicity of protoporphyrin IX metabolized from 5-aminolevulinic acid and two derivatives in human skin in vivo. Photochem Photobiol 72: 569-574, 2000.

14. Marti A, Jichlinski P, Lange N, et al: Comparison of aminolevulinic acid and hexylester aminolevulinate induced protoporphyrin IX distribution in human bladder cancer. J Urol 170: 428-432, 2003.

15. Brunner H, Hausmann F and Knuechel R: New 5-aminolevulinic acid esters - efficient protoporphyrin precursors for photodetection and photodynamic therapy. Photochem Photobiol 78: 481-486, 2003.

16. Perotti C, Fukuda H, DiVenosa G, MacRobert AJ, Batlle A and Casas A: Porphyrin synthesis from ALA derivatives for photodynamic therapy. In vitro and in vivo studies. Br J Cancer 90: 1660-1665, 2004

17. Wu SM, Ren QG, Zhou MO, Peng Q and Chen JY: Protoporphyrin IX production and its photodynamic effects on glioma cells, neuroblastoma cells and normal cerebellar granule cells in vitro with 5-aminolevulinic acid and its hexylester. Cancer Lett 200: 123-131, 2003

18. Lee JB, Choi JY, Chun JS, et al: Relationship of protoporphyrin IX synthesis to photodynamic effects by 5 -aminolaevulinic acid and its esters on various cell lines derived from the skin. Br J Dermatol 159: 61-67, 2008.

19. Moon YH, Park JH, Kim SA, Lee JB, Ahn SG and Yoon JH: Anticancer effect of photodynamic therapy with hexenyl ester of 5-aminolevulinic acid in oral squamous cell carcinoma. Head and Neck, Epub ahead of print, 2009.

20. Shrestha P, Sumitomo S, Lee CH, et al: Tenascin: growth and adhesion modulation - extracellular matrix degrading function: an in vitro study. Eur J Cancer B Oral Oncol 32B: 106-113, 1996.

21. Sinha AK, Anand S, Ortel BJ, et al: Methotrexate used in combination with aminolaevulinic acid for photodynamic killing of prostate cancer cells. Br J Cancer 95: 485-495, 2006.

22. Hosokawa Y, Shirato H, Kagei K, et al: Role of radiotherapy for mucoepidermoid carcinoma of salivary gland. Oral Oncol 35: 105-111, 1999.

23. Agulnik M and Siu LL: An update on the systemic therapy of malignant salivary gland cancers: role of chemotherapy and molecular targeted agents. Curr Med Chem Anticancer Agents 4: 543-551, 2004

24. Frimberger AE, Moore AS, Cincotta L, Cotter SM and Foley JW: Photodynamic therapy of naturally occurring tumors in animals using a novel benzophenothiazine photosensitizer. Clin Cancer Res 4: 2207-2218, 1998

25. Hsieh YJ, Wu CC, Chang CJ and Yu JS: Subcellular localization of Photofrin determines the death phenotype of human epidermoid carcinoma A431 cells triggered by photodynamic therapy: when plasma membranes are the main targets. J Cell Physiol 194: 363-375, 2003

26. Lu Z, Tao Y, Zhou Z, et al: Mitochondrial reactive oxygen species and nitric oxide-mediated cancer cell apoptosis in 2-butylamino2-demethoxyhypocrellin B photodynamic treatment. Free Radic Biol Med 41: 1590-1605, 2006.

27. Nowis D, Makowski M, Stoklosa T, Legat M, Issat T and Golab J: Direct tumor damage mechanisms of photodynamic therapy. Acta Biochim Pol 52: 339-352, 2005. 